\title{
Measurement of clinical change in progressive systemic sclerosis: a 1 year double-blind placebo- controlled trial of $\mathrm{N}$-acetylcysteine
}

\author{
D. E. FURST, P. J. CLEMENTS, R. HARRIS, M. ROSS, J. LEVY, \\ AND H. E. PAULUS \\ From the Department of Medicine, UCLA School of Medicine, Los Angeles, California, USA
}

SUMMARY $\mathrm{N}$-acetylcysteine was employed as a therapeutic agent in a 1-year, parallel, double-blindo placebo-controlled, prospective study of 22 patients with progressive systemic sclerosis (PSS). Patients were closely followed-up and were extensively evaluated at baseline, 6, and 12 month Most parameters remained unchanged during 1 year of study in both the NAC and placebo group 5 which showed the need for prolonged studies of drugs in PSS if definitive results are to be obtained? Over the year of the study the diffusing capacity decreased in both groups, while several other parac meters measured both increased and decreased. Several simple measures of function and skin dis ${ }^{\circ}$ tensibility were tested and two (oral aperature and handspread) were reproducible and sensitive enough to measure moderate changes of disease activity. They may be useful in future trials of drugs effect in PSS.

Scleroderma is a disease of unknown aetiology characterised by increased collagen deposition in the skin and fibrosis involving numerous organs (D'Angelo et al., 1969). There is also a vascular component to this disease and the immune system may be involved in the pathogenesis of some aspects of progressive systemic sclerosis (PSS) (Norton and Nardo, 1970; Johnson and Ziff, 1976; Salem and Morse, 1976; Kondo et al., 1976).

$\mathrm{N}$-acetylcysteine (NAC), as a thiol compound, is thought to act by reducing dislphide bonds of susceptible proteins to sulphydryl groups (SH). SH groups have been shown to be important in various biological phenomena, including membrane transport, preservation of the activity of enzyme systems, and cell division (Lorber et al., 1970a). Varying degrees of SH deficiency have been reported by Lorber et al. in connective tissue diseases, including scleroderma (Lorber et al., 1970a). The same report documents changes in serum SH concentration which correlate with disease activity in rheumatoid arthritis (RA). This group also showed in vitro that elevation of serum thiols results in depression of

Accepted for publication 19 September 1978

Correspondence to D. E. Furst, MD, 1000 Veteran Avenue, Los Angeles, California 90024, USA. rheumatoid factor. NAC has been shown to increase the $\mathrm{SH}$ content in soluble protein in animals and man and has been used in RA with some possible benefif (Lorber et al., 1970b; Barley and Vanal, 1970. Bailey and Sheffner, 1976). Further, acetylcystein has been shown to decrease dermal inflammatioio when given subcutaneously or orally to animals for several doses before an inflammatory agent (Baileş and Sheffner, 1976). Finally, NAC inhibits migration inhibition factor (MIF) in vitro, possibly by cleavo ing polypeptide bonds of the MIF after the MIF is produced, and NAC given intraperitoneally or oralls in large doses to rats causes suppression of in vivo in-vitro mixed lymphocyte reactions (Bartfeld and Ataynata, 1971).

\section{Patients and methods}

PSS was classified as either scleroderma or CREST (subcutaneous calcinosis, Raynaud's phenomenon $\stackrel{\rho}{\rightarrow}$ oesophageal dysmobility, sclerodactyly, telangec-0 tasis), the diagnosis being based on criteria describe $\bar{\Phi}$ previously (Clements et al., 1978). The definition of scleroderma required taut or hidebound skin beyond the face and hands, while CREST restricted taut ob hidebound skin to hands and face. The degree of 
visceral involvement was ascertained by means of a 7-10 day evaluation in hospital. Visceral involvement was defined as present in the lungs, gastrointestinal tract, cardiovascular system, and muscular system by rigorous criteria which have been outlined previously (Clements et al., 1978). Renal involvement was defined as a serum creatinine greater than 1.5.

Twenty-two PSS patients agreed to participate in the study and voluntarily gave fully informed consents (Table 1). Patients with malignant hypertension and acute renal failure were excluded from the study. Patients taking immunosuppressive drugs or prednisone (in a dose greater than $10 \mathrm{mg}$ daily) were excluded. All patients entered the study between August 1973 and August 1974. After initial evaluation to assess the extent of their disease patients were randomly assigned to NAC or placebo treated groups.

\section{MEDICATION}

Identically appearing $500 \mathrm{mg}$ capsules of NAC (supplied by Mead-Johnson, Inc.) or placebo were dispensed. Randomisation according to a random numbers table was monitored by the UCLA pharmacy. Therapy was begun with $2-4$ capsules daily and increased to a maximum of 20 capsules daily given in equally divided doses over a 2-month period. If the full dose was not tolerated, the highest tolerated dose was used.

\section{ASSESSMENTS}

On entering the study and at 6 months and 12 months ( \pm 2 months) the patients entered the UCLA Clinical Research Center for careful standard evaluations to document the degree of cutaneous and visceral involvement.

Included in the evaluation were: chest, hand, and feet radiographs, cine oesophograms, upper gastrointestinal series with small-bowel followthrough, barium enema, oesophageal manometrics, electrocardiogram, treadmill evaluations, pulmonary function tests, arterial blood gases, electromyograms

Table 1 General patient characteristics (mean + SEM)

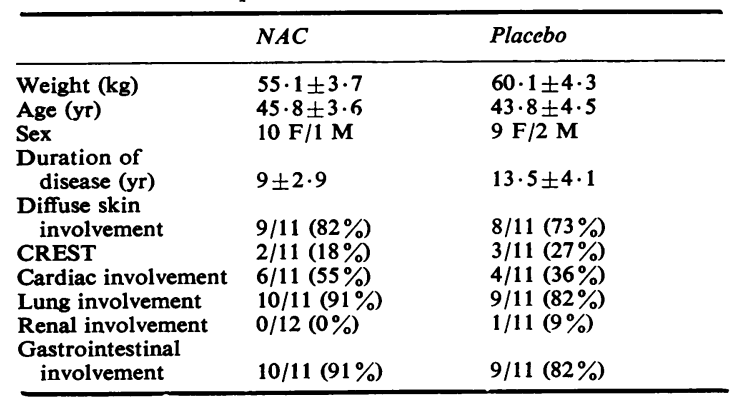

(EMG), 72-hour stool collections for faecal fat determinations, creatinine and protein determinations on a 24-hour urine collection, complete blood count (CBC) with differential and platelet count, erythrocyte sedimentation rate, quantitative immunoglobulins, serum protein electrophoresis (SPEP), anti-DNA, ANA, latex fixation, total haemolytic complement, third component of complement, aldolase, creatine phosphokinase, SMA-12 (bilirubin, alkaline phosphatase, SGOT, SGPT, total protein, calcium, phosphorus, cholesterol, glucose, BUN, uric acid, lactic dehydrogenase, serum creatinine), thyroxine, tri-iodothyronine, serum carotene. These tests were performed by standard methods by the UCLA Hospital Clinical Laboratory.

During hospitalisation and monthly thereafter the patients underwent a history and physical examination as well as a number of measures of function and skin distensibility.

Oral aperature was measured as millimeters to the outer vermillion borders of the lips when the mouth was maximally opened. The area was calculated as maximum vertical semiaxis times maximal horizontal semiaxis times $\pi$ (area of an ellipse).

Handspread was measured as the maximum distance in millimeters from tip of thumb to most protruding portion of the fifth digit (usually the tip of the finger) when the patient spread her hand, palm down, on the table (no downward pressure was to be applied-only lateral stretch).

The distance between the tip of the third finger and the most distal observable flexor wrist crease was measured in millimeters as the patient tried to touch her wrist with her third finger.

Grip strength was the highest pressure achieved on a sphygmomanometer for a sustained period on the second or third of 3 attempts with each hand, as outlined by Lee et al. (1974). The sphygmomanometer was used wrapped in a standard manner and was used only for this purpose. Baseline pressure was $40 \mathrm{mmHg}$. The patient was asked to hold her arm unsupported and palm up. If this was not possible, then the same position was used at each visit.

Ring size of the proximal interphalangeal of the third finger of each hand was measured with jeweller's rings.

Joint count was the sum of tender joints as assessed by firm palpation of 29 joints or groups of joints as previously published (Furst et al., 1977).

Statistical analysis was by Student's paired and unpaired $t$ tests.

\section{Results}

From Tables 1 and 2, it can be seen that these patients were comparable at the initiation of the study. Of the 
Table 2 Baseline values and changes over 1 year (mean $\pm S E M)$

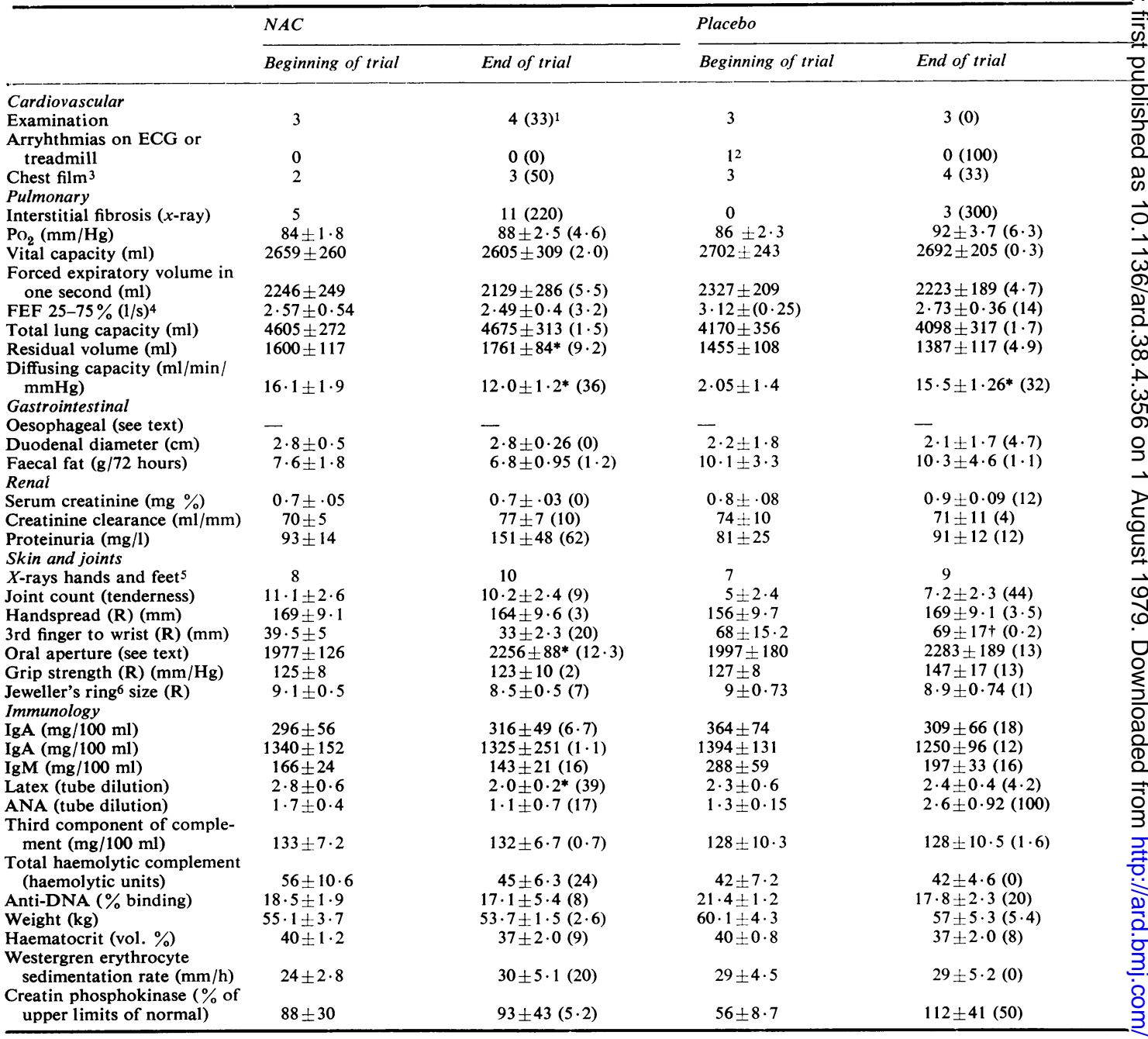

1 Percentage change between beginning and end. ${ }^{2}$ Recurrent paroxysmal atrial tachycardia (PAT). ${ }^{3}$ Cardiac enlargement or pulmonary arteryo prominence. 4 Forced expiratory flow 25 to $75 \%$. 5Tuft resorption and/or subcutaneous calcinosis. ${ }^{6} \mathrm{Measured}$ over 3 rd PIP. ${ }^{* W}$ ithin group statistical difference $\mathrm{P}<0.05$. † Between group statistical sifference $\mathrm{P}<0.05$.

11 patients who started on NAC 4 discontinued medication: 2 secondary to gastrointestinal distress, 1 secondary to a rash, and 1 because of deterioration in disease. The average daily dose of drug for the 7 who completed the 1 -year study was $9 \cdot 4 \pm 0.4 \mathrm{~g}$ (SEM). Four of the 11 placebo treated patients discontinued medication: 2 because their disease deteriorated, 1 secondary to gastrointestinal distress, and 1 because she did not wish to continue to take so many capsules. Average daily dose for the placebotreated'group was $9 \cdot 7 \pm 0 \cdot 2 \mathrm{~g}$. Thus the comparison at 1 year was actually between 7 NAC-treated patients and 7 placebo-treated patients.
The results in each area of interest are included in. Table 2. There were no significant changes when $\tilde{O}$ comparing the 2 groups over the year of study N except in testing the distance from the tip of the 3rdo finger to the wrist crease. This difference is related too the large (although not statistically significant) difference between the 2 groups at baseline. Each $\stackrel{\mathcal{C}}{+}$ group had significant $(\mathrm{P}<0 \cdot 02)$ deterioration in diffusing capacity in 1 year. In the NAC-treated group residual volume increased significantly $(\mathrm{P}<0 \cdot 05)$. Oral aperature increased significantly in $\mathbb{8}$ the NAC-treated group $(P<0 \cdot 01)$ and latex titres decreased statistically significantly $(P<0 \cdot 05)$. 
The muscle testing parameters employed (CPK, aldolase, biopsy, EMG) were those which have been published previously (Clements et al., 1978). They did not change significantly in either group over the year of study.

Osophageal manometrics and cine oesophograms were also analysed separately, and they too remained unchanged during the study for both the placebo and NAC-treated groups.

Complete blood count, platelet count, differential, SMA-12, thyroxine, tri-iodothyronine, arterial $\mathrm{PCO}_{2}$, and base excess as well as some of the remaining pulmonary function tests were not included in Table 2 as they did not change and added nothing to the information presented in the Table.

Oral aperature was evaluated as a measure of skin distensibility. The area measured was calculated as an ellipse, as the open mouth is more ellipsoid than circular. In 10 normal volunteers this area was $3076 \pm 167$ (SEM) $\mathrm{mm}^{2}$. Mean intraobserver differences, measured in 10 PSS patients by the same observer at approximately the same time of day for 3 consecutive days, was $4 \%$. Interobserver differences were $19 \%$ and diurnal variation was $15 \%$ (see Table $3)$. The coefficient of variation (CV), defined as the mean divided by the standard deviation, averaged $0 \cdot 24$. This is a measure of relative variability and gives an idea of the precision of the measurement. A low CV (e.g., 0.05) indicates that the measurements are very reproducible, while a high $\mathrm{CV}$ (e.g., $0 \cdot 50$ ) shows that there is much variation about the mean. A CV of 0.27 indicates moderate relative variability and indicates that a moderate change in disease state must occur before the measurement in question can reliably detect it.

Handspread measures were surprisingly consistent between observers (differences were 1 to $5 \%$ ), but diurnal variation was large (about 16\%) (Table 3). Coefficients of variation averaged 0.25 .

The most inconsistent measures were those relating to the distance from the tip of the third finger to the most distal wrist crease. Here coefficients of variation averaged $0 \cdot 52$. It appeared as if evening measurements were slightly more consistent than morning ones, perhaps a sign of less gel phenomenon in the hands by evening time (Table 3). The timing of measurements was as for oral aperature.

As can be seen in Table 2, changes in both groups were generally in the same direction over the year of study. For the NAC-treated group the greatest percentage changes occurred in the diffusing capacity (Dco), latex titre, and proteinuria. The change in Dco, along with increased residual volume, indicated statistical worsening of disease but may not be clinically significant during the period of the study. Oral aperture increased significantly. Latex titre decreased statistically, although not clinically, significantly. This latter change would be expected on the basis of previous reports using NAC (Lorber et al., 1970b).

There were no significant changes in any of the parameters examined in the placebo-treated group, except for Dco, where significant deterioration was noted.

\section{Discussion}

This 1-year double-blind randomised placebocontrolled study of NAC in the treatment of PSS was discontinued after 22 patients had been entered into the trial because additional supplies of NAC and its matched placebo were no longer available.

When the data was analysed after all entered patients had continued medication for a year (or had

Table 3 Variation in measures of skin distensibility

\begin{tabular}{|c|c|c|c|c|c|c|}
\hline & \multirow[t]{2}{*}{$N$} & \multirow[t]{2}{*}{$\begin{array}{l}\text { Oral aperture } \\
\left(\mathrm{mm}^{2}\right)\end{array}$} & \multicolumn{2}{|l|}{ Handspread $(\mathrm{mm})$} & \multicolumn{2}{|c|}{$\begin{array}{l}\text { Tips of third finger to wrist } \\
\text { crease }(\mathrm{mm})\end{array}$} \\
\hline & & & $\boldsymbol{R}$ & $\boldsymbol{L}$ & $\boldsymbol{R}$ & $L$ \\
\hline \multicolumn{7}{|l|}{ Measured by D.E.F. in a.m. } \\
\hline 1st & 10 & $1938 \pm 441 *(0 \cdot 23) \dagger$ & $156 \pm 37(0 \cdot 23)$ & $162 \pm 46(0 \cdot 28)$ & $35 \pm 25(0.71)$ & $29 \pm 14(0.48)$ \\
\hline 2nd & 10 & $1997 \pm 634(0 \cdot 32)$ & $163 \pm 40(0 \cdot 24)$ & $168 \pm 47(0 \cdot 28)$ & $34 \pm 30(0.88)$ & $40 \pm 17(0.43)$ \\
\hline 3rd & 10 & $2014 \pm 570(0 \cdot 28)$ & $166 \pm 42(0 \cdot 25)$ & $170 \pm 46(0.27)$ & $36 \pm 26(0.72)$ & $38 \pm 16(0.42)$ \\
\hline \multicolumn{7}{|c|}{$\begin{array}{l}\text { Maximum difference in } \\
\text { measures made by D.E.F. }\end{array}$} \\
\hline $\begin{array}{l}\text { measures made by D.E.F. } \\
\text { in a.m. }\end{array}$ & 10 & $76(4 \%) \S$ & $13(8 \%)$ & $8(5 \%)$ & $2(6 \%)$ & $11(28 \%)$ \\
\hline $\begin{array}{l}\text { Measures by D.E.F. in p.m. } \\
\text { Maximum difference for }\end{array}$ & 6 & $2430 \pm 636(0 \cdot 26)$ & $181 \pm 37(0 \cdot 20)$ & $193 \pm 25(0 \cdot 13)$ & $37 \pm 10(0 \cdot 27)$ & $35 \pm 17(0.49)$ \\
\hline \multirow{3}{*}{$\begin{array}{l}\text { D.E.F. a.m. vs. p.m. } \\
\text { Measured by P.J.C. in p.m. } \\
\text { Differences between D.E.F. } \\
\text { and P.J.C. in p.m. }\end{array}$} & $\begin{array}{r}10 \\
6\end{array}$ & $\begin{array}{l}291(15 \%) \\
2013+536(0.27)\end{array}$ & $\begin{array}{l}25(15 \%) \\
179+30(0 \cdot 17)\end{array}$ & $\begin{array}{c}31(17 \%) \\
183+30(0.16)\end{array}$ & $\begin{array}{l}3(8 \%) \\
29+9(0.31)\end{array}$ & $\begin{array}{l}6(17 \%) \\
27+12(0.44)\end{array}$ \\
\hline & & & & & & \\
\hline & 10 & $417(19 \%)$ & $2(1 \%)$ & $10(5 \%)$ & $8(24 \%)$ & $8(29 \%)$ \\
\hline
\end{tabular}

*Standard deviation. †Coefficient of variation. §Percentage difference. D.E.F. and P.J.C. are the investigators' initials. $R=$ right; $\mathbf{L}=$ left.

a.m. = between 7 a.m. and 10 a.m.; p.m. = between 4 p.m. and 7 p.m. 
discontinued medication early), we became aware of several facts. We had initiated the study for the dual purpose of evaluating NAC as a treatment modality in PSS and in order to study intensively the natural history of cutaneous and visceral involvement in PSS. We confirmed the high frequency of abnormal Dco in PSS lung disease, as had been noted previously by Guttadauria et al. (1977). We also noted decreased total lung capacities and FEF $25-75 \%$. We found, however, that Dco changed more than the other pulmonary function tests and may therefore be a more sensitive measure of pulmonary function change in our group of patients, as has been suggested previously (Wilson et al., 1964).

The degree of muscle involvement was surprising $(90 \%)$ and is detailed in another publication. Further, the recurrent evaluations initiated with this study have pointed out some of the spontaneous serological and serum changes that occur in PSS. ANA was positive $(>1: 10)$ in $6(27 \%)$ patients at initial evaluation. Over the year of study 1 further patient became positive, while most of the other 6 who were originally positive had significant titre changes (Table 4). Latex was positive in $5(23 \%)$ initially, and became positive in 1 more, while 4 patients had significant drops in these latex titres (Table 4). CPK, too, changed by large amounts (Table 4).

Methods to evaluate skin distensibility and tethering have been examined in the past: for example, that published by Grahame and Harvey (1975). The manoeuvres tend to be rather cumbersome and difficult to use, however. We therefore tested several simplified measures of skin distensibility, although it is realised that those which we used are to some extent a combined measure of joint, muscle, and skin function. In order to evaluate the variability of these measurements 10 patients were examined on 3 successsive days while in hospital for evaluation.

For oral aperture there was no correlation of the area of the oral aperture with temperomandibular pain or joint count. As can be seen in Table 3, there was little variation in measurement of mean oral aperture when 1 observer did all the measurements

Table 4 Changes in parameters over 1 year

\begin{tabular}{|c|c|c|c|c|}
\hline & \multicolumn{2}{|l|}{6 months } & \multicolumn{2}{|l|}{12 months } \\
\hline & Rises & Declines & Rises & Declines \\
\hline $\begin{array}{l}\text { DCo } \ddagger \\
\text { ANA* } \\
\text { Latex** } \\
\text { CPK } \dagger\end{array}$ & $\begin{array}{l}\overline{2}(9 \%) \\
\overline{1}(4 \cdot 5 \%)\end{array}$ & $\begin{array}{l}10(45 \%) \\
\frac{1}{3(14 \%)} \\
3(14 \%)\end{array}$ & $\begin{array}{l}1(4 \cdot 5 \%) \\
3(14 \%) \\
2(9 \%) \\
4(19 \%)\end{array}$ & $\begin{array}{r}14(67 \%) \\
4(18 \%) \\
4(18 \%) \\
4(19 \%)\end{array}$ \\
\hline
\end{tabular}

Number in parentheses is the percentage of patients who changed. $\mp$ Change of $4 \mathrm{ml} / \mathrm{min} / \mathrm{mmHg}$ definied as a real change.

*Change of 2 tube dilutions defined as a real change.

tChange of $50 \%$ from beginning of study defined as a real change. at approximately the same time of day, while diurnalo variation and interobserver errors both contributed significantly to variability. The coefficient of variation of about $\mathbf{0 . 2 7}$ indicates that the test is only moder-을 ately sensitive as an indicator of disease change.

The degree of contracture of the fingers, pain due $\frac{\bar{\sigma}}{\widetilde{D}}$ to ulceration, or joint pathology and bone resorp- $\stackrel{\varnothing}{\varrho}$ tion would all be expected to affect the measurement of hand spread. However, within a given individualthese changes would be minimised over a short ${ }_{-}^{\circ}$ period and the reproducibility of the measurement $\vec{\omega}$ could be assessed. The mean intraobserver error and interobserver differences were both approximately $5 \%$. Like oral aperture, the CV was about $0 \cdot 25 . \omega_{\infty}$ Since the maximum mean differences are relatively small, and are consistent from morning to evening, $\omega$ this measurement may be useful as a measure ofo moderate changes in this disease.

The distance from the tip of the third finger tothe most distal wrist creases was occasionally? impossible to ascertain because no wrist crease coulde

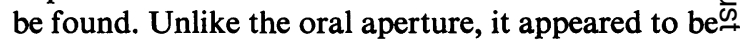
greatly influenced by joint stiffness, effort, ulcers, $\vec{\theta}$ occasional difficulty in finding the wrist crease, etc. It was a much less consistent measure than the others, as is shown by the large CV for this measure (average: $0 \cdot 52$ ) (Table 3 ) and probably is not a very useful measure.

Unfortunately there is no accepted effective therapy in PSS, so that it is impossible to demonstrate the sensitivity of these techniques as measureso응 of drug effect. At present they should be viewed as simple, rapid methods to measure the presence of changes in skin, joint, and muscle in PSS. Oral aperture may be a more 'pure' measure of skin distensibility than the other tests as it appears to beo least affected by alterations in the joint. It may nevertheless reflect muscular or tendinous as well as? skin changes.

Of central interest to this report is the findingo that there was no significant change in most of the parameters measured in the placebo group over the year of study. This emphasises the fact that PSS is in general a disease in which measurable changes occuros over long periods of time. The lack of change in our placebo group is in part secondary to the lack of $\mathbb{E}^{-}$ sensitivity of the measurements available to ascertaino change in PSS and in part because the disease ofteno does change slowly. Our patients, although having extensive visceral involvement, had had their disease? for long periods and were not changing rapidly. Further, the drug we used was known to be benigno and without serious toxicity. This perhaps introduced a patient-selection bias towards patients withoutक severe, rapidly progressive disease. Finally, a majoro pathological feature of PSS is fibrosis, which may be 
irreversible. We might therefore have expected our patients to stabilise with effective therapy rather than to show significant improvement. For all the above reasons we feel that it is generally necessary to continue studies of PSS for longer than 1 year unless a group of patients with rapidly progressive disease is chosen for the study.

Since the above factors are operative in many of the reported studies (Jansen et al., 1968; Silver and Gitlin, 1973; D'Alacron-Sergovia et al., 1974; Steigerwald, 1974; Binnick et al., 1977). we feel that other published studies of 1 year's duration or less, even if controlled, may not have been conclusive on the basis of the length of the study alone.

One year of NAC therapy produced no significant improvement in the disease. Unfortunately, because we were not able to continue the drug for more than 1 year and there was no significant deterioration in the placebo-treated patients we cannot determine whether NAC prevented progression of the disease.

We gratefully acknowledge the dedicated help of the UCLA Clinical Research Center staff during the course of this study and the secretarial assistance of Evelyn M. Tackels.

This study was supported in part by USPHS grants GM 15759 and RR 865.

\section{References}

Bailey, K. R., and Sheffner, A. L. (1976). The reduction of experimentally induced inflammation by sulfhydryl compounds. Biochemical Pharmacology, 16, 1175-1182.

Barley, J. P., and Vanal, M. E. (1970). Acetylcysteine in the treatment of rheumatoid arthritis. Unpublished study Courtesy of Mead-Johnson Research Center.

Bartfeld, H., and Ataynata, T. (1971). N-acetylcysteine inactivates migration inhibition factor and delayed hypersensitivity reactions. Nature New Biology, 231, 157158.

Binnick, S. A., Shore, S. S., Corman, A., and Fleischmeyer, R. (1977). Failure of dimethyle sulfoxide in the treatment of scleroderma. Archives of Dermatology, 1113, 13981402.

Clements, P. J., Furst, D. E., Campion, D. S., Bohan, A., Harris, R., Levy, J., and Paulus, H. E. (1978). Muscle disease in progressive systemic sclerosis. Arthritis and Rheumatism, 21, 62-71.
D'Alacron-Sergovia, Ibanez, G., and Kershenobich, D. (1974). Treatment of scleroderma. Lancet, 2, 1054-1055.

D'Angelo, W. A., Fries, J. F., Masi, A. T., and Shulman, L. E. (1969). Pathologic observations in systemic sclerosis (scleroderma). American Journal of Medicine, 46, 428-440.

Furst, D. E., Levine, S., Srinivasin, R., Metzger, A. L. Bangert, R., and Paulus, H. E. (1977). A double-blind trial of high versus conventional dosages of gold salts for rheumatoid arthritis. Arthritis and Rheumatism, 20, 1473-1480.

Grahame, R., and Harvey, W. (1975). Cutaneous extensibility in health and disease. Rheumatism and Rehabilitation, 14, 87-99.

Guttadauria, M., Ellman, H., Emmanuel, G., Kaplan, D., and Diamond, H. (1977). Pulmonary function in scleroderma. Arthritis and Rheumatism, 20, 1071-1079.

Jansen, G. T., Barraza, D. E., Ballard, J. L., Honeycutt, W. N., and Dillaha, C. J. (1968). Generalised scleroderma treatment with an immunosuppressive agent. Archives of Dermatology, 97, 690-698.

Johnson, R. L., and Ziff, M. (1976). Lymphokine stimulation of collagen accumulation. Journal of Clinical Investigation, 58, 240-252.

Kondo, H., Robin, B. S., and Rodnan, G. P. (1976). Cutaneous antigen stimulating lymphokine production by lymphocytes of patients with progressive systemic sclerosis (scleroderma). Journal of Clinical Investigation. 58, 13881394.

Lee, P., Baxter, A., Dick, W. C., and Webb, J. (1974). An assessment of grip strength measurement in rheumatoid arthritis. Scandinavian Journal of Rheumatology, 3, 17-23.

Lorber, A., Bocy, R. A., and Chang, C. C. (1970). Sulf hydryl deficiency in connective tissue disorders: correlation with disease activity and protein alterations. Metabolism, 20, 446-455.

Lorber, A., Chang, C. C., Masuoka, D., and Meacham, I. (1970). Effect of thiols in biological systems on protein sulphydryl content. Biochemical Pharmacology, 19, 1551.

Norton, W. L., and Nardo, J. M. (1970). Vascular disease in progressive systemic sclerosis (scleroderma). Annals of Internal Medicine, 73, 317-324.

Salem, N. B., and Morse, J. H. (1976). Lymphocyte response to mitogens in progressive systemic sclerosis. Arthritis and Rheumatism, 19, 875-882.

Silver, W., and Gitlin, N. (1973). Progressive systemic sclerosis (diffuse scleroderma): a follow-up report of treatment with potaba. American Medical Journal, 2 , 1001-1002.

Steigerwald, J. C. (1974). Chlorambucil therapy in progressive rheumatism. Arthritis and Rheumatism. Suppl. 1, 74.

Wilson, R. J., Rodnan G. P., and Robin, E. D. (1964). An early pulmonary physiologic abnormality in progressive systemic sclerosis (diffuse scleroderma). American Journal of Medicine, 36, 361-363. 